\title{
Estimation des erreurs d'étalonnage de thermomètres infrarouges industriels liés à la méconnaissance de l'émissivité de sources et des bandes spectrales de thermomètres infrarouges
}

\author{
Olga Kozlova ${ }^{1, a}$, Lydia Rongione ${ }^{1}$ et Stéphan Briaudeau ${ }^{1}$ \\ ${ }^{1}$ Laboratoire Commun de Métrologie LNE-CNAM, 61 rue du Landy, 93210 La Plaine Saint Denis, France
}

\begin{abstract}
Usually, NMIs propose calibration services of blackbody sources or infrared thermometer in a specific spectral band. Due to the use of the specific transfer infrared thermometer such a calibration is valid only for this specific spectral band and for specific infrared thermometer spectral sensitivity. In this paper we will focus on the errors introduced by the calibration process in the transfer laboratory due to the different spectral response of the industrial infrared thermometer under calibration and the specific transfer infrared thermometer used for the blackbody calibration. The magnitude of this error depends both on the difference in spectral responsivities of the transfer and industrial infrared thermometers and on the spectral emissivity of the transfer blackbody used. Most frequently, information about the spectral response of the infrared thermometer and the spectral emissivity of the blackbody are not available. Moreover the measurement of the spectral responsivity of the infrared thermometer and the spectral emissivity of the blackbody source is way too complicated and time consuming to be performed. In this paper, we present the results of the estimation of this error for different typical cases, which could be of interest for the transfer laboratories to establish their uncertainty budget as well as the possible effects on the uncertainties of calibration of industrial infrared thermometer for end-users.
\end{abstract}

\section{Introduction}

Les méthodes de mesure de température sans contact sont largement utilisées pour le suivi des processus de production ou de traitement du plastique, du verre, des métaux, des composants électroniques, du papier, pour la maintenance générale dans les secteurs du transport, de l'électricité ou de la climatisation, dans les processus de découpe et de soudage au laser ou encore pour le contrôle sanitaire des produits alimentaires, etc.

Pour la plage de températures entre $-20^{\circ} \mathrm{C}$ et $600^{\circ} \mathrm{C}$, et pour les surfaces usuelles (non-métalliques ou métalliques et revêtus), les thermomètres infrarouges fonctionnant dans la bande spectrale dite $« 8-14 \mu \mathrm{m}$ » sont les plus adaptés.

Ces appareils disposent d'un détecteur thermique de type thermopile ou pyro-électrique, qui ont une sensibilité spectrale constante sur toute la plage d'utilisation $(2-40$ $\mu \mathrm{m})$. Le filtre placé devant le détecteur sélectionne la bande spectrale de fonctionnement. C'est la transmission spectrale du filtre et la transmission spectrale des éléments optiques qui déterminent la sensibilité spectrale du thermomètre infrarouge.

La largeur de bande et la sensibilité spectrale peuvent fortement varier d'un thermomètre infrarouge à l'autre. La plupart du temps, les fabricants de thermomètres industriels indiquent uniquement que l'appareil fonctionne en bande $8-14 \mu \mathrm{m}$ sans autre information précise sur la sensibilité spectrale. Cependant, lors de l'étalonnage de ces thermomètres infrarouges la différence de sensibilité spectrale entre le thermomètre étalon et celui à étalonner peut engendrer un biais. Ce biais est d'autant plus grand que l'émissivité de la source s'écarte de la valeur unitaire.

Dans cet article nous nous focaliserons sur les erreurs d'étalonnage liées à la sensibilité spectrale des thermomètres infrarouges et l'émissivité des sources utilisées pour les étalonner.

\section{Explication de la méthode}

\subsection{Processus d'étalonnage}

Parmi d'autres nombreuses prestations, le LNE propose l'étalonnage de thermomètres infrarouges et de sources planes. Ci-dessous nous donnons un bref aperçu de principe général de l'étalonnage de sources planes et de thermomètres infrarouges en bande $8-14 \mu \mathrm{m}$ et des limites de validité de la méthode d'étalonnage.

Pour étalonner une source plane, cette source est comparée à la source de référence grâce à un

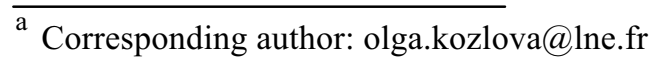


thermomètre infrarouge de transfert. La source de référence est une cavité corps noir de très haute émissivité parfaitement caractérisée. A l'issue de la comparaison de source plane et de source de référence, une température de luminance ${ }^{1}$ est affectée à la source en question.

Une source ainsi étalonnée peut ensuite être utilisée par des laboratoires de transfert pour effectuer des étalonnages de thermomètres infrarouges industriels fonctionnant dans la même bande spectrale que celle de l'étalonnage. Cependant, si les thermomètres infrarouges industriels n'ont pas la même sensibilité spectrale que le thermomètre infrarouge de transfert qui a servi à étalonner la source, les biais d'étalonnage sont possibles. Ces biais sont d'autant plus importants que les différences des sensibilités spectrales sont grandes et que l'émissivité de la source étalonnée s'écarte de la valeur unitaire.

Deux méthodes peuvent être utilisées au LNE pour l'étalonnage des thermomètres infrarouges :

- la méthode «directe» consiste à viser une source de référence dont la température de luminance est parfaitement connue dans la bande spectrale de fonctionnement du thermomètre infrarouge à étalonner ;

- la méthode «par comparaison» utilise un thermomètre infrarouge de référence. La réponse $\mathrm{du}$ thermomètre infrarouge à étalonner est comparée (en termes de température de luminance) à celle du thermomètre infrarouge de référence face à une source de haute émissivité.

Le thermomètre infrarouge étalonné peut être ensuite utilisé par un laboratoire de transfert comme un thermomètre infrarouge de transfert pour effectuer des étalonnages des thermomètres infrarouges industriels. Dans le cas idéal, thermomètre infrarouge de référence doit avoir la même sensibilité spectrale que celle des thermomètres infrarouges à étalonner.

Quelle que soit la méthode d'étalonnage utilisée et l'appareil étalonné (source ou thermomètre infrarouge), son étalonnage doit respecter certaines conditions :

- équivalence des bandes spectrales des thermomètres ;

- $\quad$ sources de grande émissivité et d'émissivité grise.

Le but de cet article est de présenter une estimation des biais liés aux différences de sensibilités spectrales et/ou aux différences d'émissivités spectrales des sources.

\subsection{Méthode de calculs}

Pour estimer un biais causé par la différence de bandes spectrales des thermomètres infrarouges face à une source d'émissivité non unitaire, la méthode décrite cidessous a été utilisée.

\footnotetext{
${ }^{1}$ La température de luminance d'une source (ou température équivalente corps noir) est la température du corps noir qui aurait la même luminance spectrale que la source.
}

Connaissant la sensibilité spectrale d'un thermomètre infrarouge $S(\lambda)$ positionné dans un environnement à la température $T_{e n v}=23^{\circ} \mathrm{C}$ et face à une source d'émissivité $\varepsilon(\lambda)$ et de température de surface $T$, nous pouvons calculer la luminance $L$ reçu par le détecteur (flux par unité de surface émissive et unité de l'angle solide dans une direction choisie). Cette luminance $L$ est constituée de la luminance qui provient de source $L_{\text {source }}$ et de la luminance de l'environnement réfléchi par la source $L_{e n v}$ :

$$
\begin{gathered}
L=L_{\text {source }}+L_{\text {env }} \\
L_{\text {source }}=\int S(\lambda) \cdot \varepsilon(\lambda) \cdot L_{\lambda}(T) \cdot d \lambda \\
L_{\text {env }}=\int S(\lambda) \cdot(1-\varepsilon(\lambda)) \cdot L_{\lambda}\left(T_{\text {env }}\right) \cdot d \lambda
\end{gathered}
$$

où $L_{\lambda}$ est la densité spectrale de luminance (luminance spectrique) à la température $T$ et à la longueur d'onde $\lambda$. La luminance spectrique est donnée par la loi de Planck:

$$
L_{\lambda}(T)=\frac{c_{1 L}}{\lambda^{5}} \cdot\left[\exp \left(\frac{c_{2}}{\lambda \cdot T}\right)-1\right]^{-1}
$$

où $c_{1 L}$ et $c_{2}$ sont les constantes de rayonnement, de valeurs ${ }^{2}: c_{1 L}=1,191042953(15) \times 10^{-16} \mathrm{~W} \cdot \mathrm{m}^{2} \cdot \mathrm{sr}^{-1}$ et $c_{2}=$ $1,43877736(83) \mathrm{m} \cdot \mathrm{K} ; \lambda$ est la longueur d'onde de rayonnement et $T$ la température thermodynamique de la source de rayonnement.

Notons que nous ne considérons pas le signal en sortie du détecteur, mais la luminance de la source reçue par détecteur. Le signal en sortie du détecteur est proportionnel au flux reçu par le détecteur à une constante $C$ près :

$$
\text { Signal }=C \cdot L
$$

La constante $C$ dépend du gain de conversion de l'électronique et du facteur de forme des optiques qui définit la surface et l'angle solide de visée, qui sont spécifiques pour chaque thermomètre infrarouge. Pour la simplicité et sans restreindre le modèle, nous poserons que la constante $C$ est égale à 1 .

Par ailleurs, le thermomètre infrarouge a sa propre sensibilité thermique, définie comme la dépendance du signal en sortie du détecteur en fonction de la température de la source Signal $_{\text {etal }}=f(T)$. Pour connaître cette sensibilité thermique l'appareil est étalonné à plusieurs températures face à une source corps noir à l'émissivité unitaire. Le signal du thermomètre est alors modélisé comme suit (avec $C=1$ et $\varepsilon(\lambda)=1$ ):

$$
\text { Signal }_{\text {etal }}=f(T)=1 \cdot \int S(\lambda) \cdot 1 \cdot L_{\lambda}(T) \cdot d \lambda
$$

Rappelons que pour le corps noir d'émissivité unitaire, la température de surface de la source $T$ est égale à la température de luminance $T_{\text {lum }}$. On peut donc, utiliser la dépendance inverse $T=T_{\text {lum }}=f^{\prime}\left(\right.$ Signal $\left._{\text {etal }}\right)$ comme une courbe d'étalonnage pour calculer la température de luminance qui correspond au signal mesuré ${ }^{3}$. Pour

\footnotetext{
2014 CODATA

${ }^{3}$ Notons que cette modélisation correspond au processus réel d'étalonnage d'un thermomètre infrarouge face à une source corps noir : on attribue une température de luminance à une
} 
interpoler la courbe entre les points d'étalonnage nous ajustons un modèle phénoménologique (Figure 1). Pour un modèle phénoménologique nous utilisons une fonction suivante [2-4]:

$$
T_{\text {lum }}=f^{\prime}(\text { Signal })=\frac{1}{b \cdot \ln (1+a / \text { Signal })}+c
$$

où $a, b$ et $c$ sont les paramètres d'ajustement.

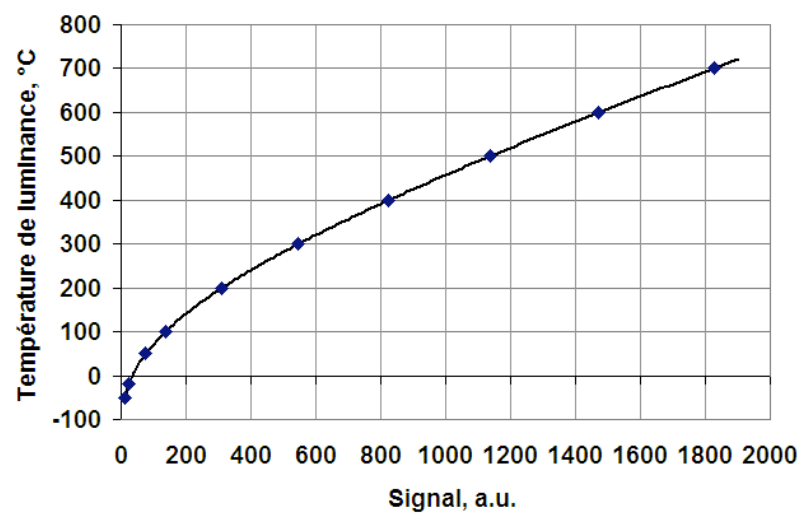

Figure 1. Exemple d'une courbe d'étalonnage $T_{\text {lum }}=f^{\prime}$ (Signal). Points bleus : points d'étalonnage calculés avec l'équation (6). Courbe noire : ajustement avec un modèle phénoménologique (équation (7)). L'écart maximal entre les points et le modèle est inferieur à $0.06^{\circ} \mathrm{C}$.

Avec un tel d'étalonnage nous pouvons calculer la température de luminance pour une source d'émissivité arbitraire. Pour calculer la différence de température de luminance qui seraient mesurées par des thermomètres infrarouges de sensibilités spectrales $S(\lambda)$ différentes positionnés face à un corps noir de température $T$ et d'émissivité $\varepsilon(\lambda)$, nous avons choisi des représentations simplifiées de courbes de sensibilité et d'émissivité spectrales pour lesquelles nous avons réitéré les calculs suivants :

- pour chaque sensibilité $S_{x}(\lambda)$, le signal théorique Signal du thermomètre face à une source d'émissivité $\varepsilon_{x}(\lambda)$ est calculé à l'aide des équations (1) - (5) ;

- la température de luminance correspondante est déduite de la courbe d'étalonnage théorique du thermomètre de sensibilité $S_{x}(\lambda)$ face à un corps noir (équation (7)) ;

- les valeurs de la température de luminance obtenue sont comparées pour les thermomètres infrarouges avec les différentes sensibilités spectrales.

\section{Résultats des calculs}

Ce paragraphe présente les résultats des calculs de température de luminance de sources d'émissivités différentes affectée par des thermomètres infrarouges de différentes sensibilités spectrales.

Dans la première partie nous allons nous focaliser sur l'influence de la forme de la dépendance spectrale de la

réponse de thermomètre infrarouge. Ensuite le thermomètre infrarouge est utilisé pour mesurer la température de luminance d'une source quel qu'on que. sensibilité du thermomètre infrarouge et de la forme spectrale de l'émissivité de la source sur la température de luminance mesurée, en limitant strictement la largeur de bande à $8-14 \mu \mathrm{m}$.

Dans la deuxième partie, nous allons calculer l'influence de la largeur de bande et du décalage de la bande spectrale du thermomètre infrarouge face à des sources d'émissivité différentes sur la température de luminance.

\subsection{Influence de la forme}

Pour estimer les différences de température de luminance mesurée par les thermomètres infrarouges face à une source nous avons choisi plusieurs types de dépendance des sensibilités spectrales des thermomètres. Nous avons également fait varier l'émissivité spectrale de sources.

Pour la sensibilité spectrale les 5 formes schématiques ont été choisies (Figure 2). Notons que la sensibilité spectrale S1 d'allure uniforme rectangulaire correspond à la sensibilité idéale. Les écarts de température de luminance sont donc calculés par rapport aux valeurs obtenues pour un thermomètre infrarouge qui aurait une telle sensibilité.

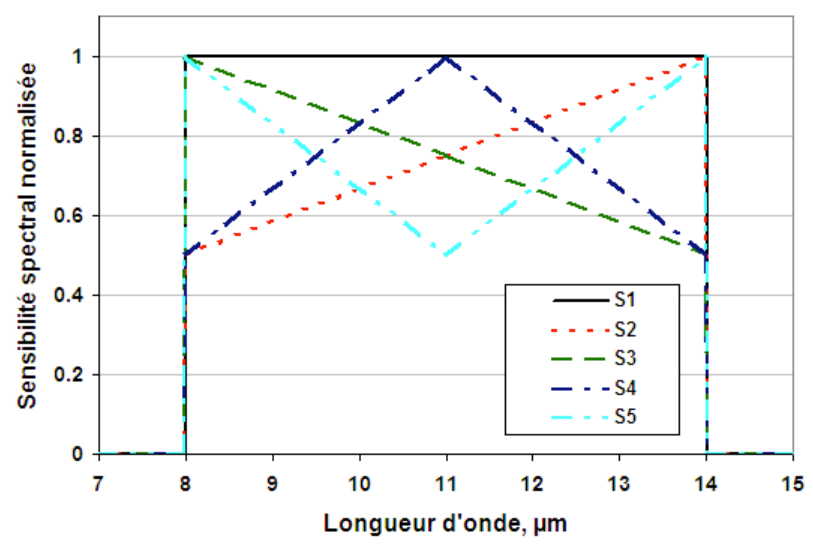

Figure 2. Différents types de sensibilité spectrale des thermomètres infrarouges (dans la bande $8-14 \mu \mathrm{m}$ ) utilisés pour les calculs.

Les limites de variation de l'émissivité sont choisies entre 0,92 et 1 , proches de valeurs typiques des constructeurs de sources planes (Figure 3, émissivités E1 - E4). Pour les sources non grises, nous avons sélectionné 4 formes schématiques de variation spectrale de l'émissivité dans la limite des valeurs typiques (Figure 3, émissivités E5 - E8).

Notons que émissivité E1 correspond au corps noir idéal. Dans ce cas, la température luminance est égale à la température de la source et les résultats sont indépendants de la sensibilité spectrale des thermomètres infrarouges. 


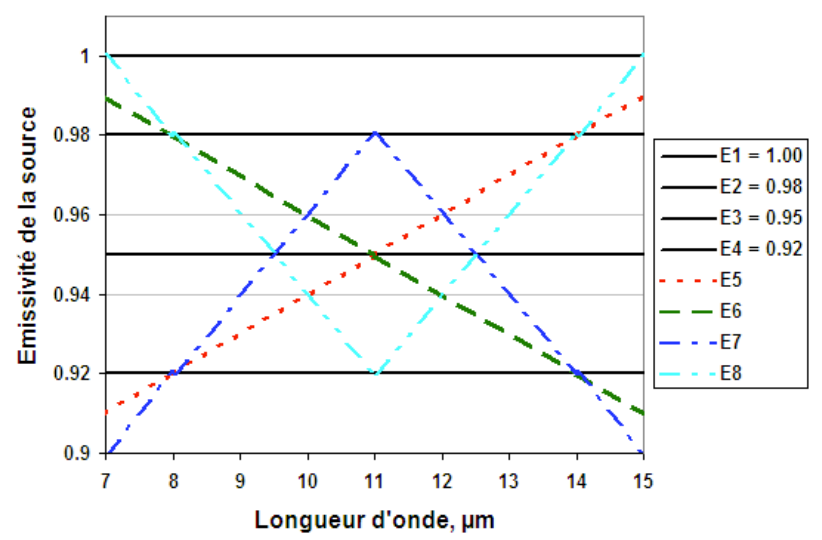

Figure 3. Différents types d'émissivité de la source utilisés pour les calculs.

Le tableau 1 montre les écarts de températures de luminance qui seraient mesurées avec des thermomètres infrarouges de sensibilité S2 à S5 et face à des sources d'émissivités E1 à E8 par rapport à celles mesurées par le thermomètre infrarouge de sensibilité $\mathrm{S} 1$ face aux mêmes sources. Ces écarts de température peuvent être supérieurs à $0,5^{\circ} \mathrm{C}$ (marqués en rouge dans le tableau 1) pour les températures élevées et pour les émissivités nonconstantes.

Notons que les écarts de température de luminance obtenus face à un corps noir idéal (E1) sont dus à la méthode de calculs, et plus exactement, au modèle phénoménologique $T_{\text {lum }}=f^{\prime}$ (Signal) utilisé (Equation (7)).

\subsection{Influence de la bande}

Dans ce paragraphe nous nous focalisons sur l'influence de la bande spectrale du thermomètre infrarouge sur les mesures de la température de luminance de la source de l'émissivité non-unitaire. Plusieurs variations de largeurs de bandes spectrales par rapport à la bande idéale $8-14$ $\mu \mathrm{m}(\mathrm{S} 1)$ ont été étudiées:

- décalage de bande spectrale avec conservation de la largeur totale de la bande : bandes $7-13$ $\mu \mathrm{m}$ et $9-15 \mu \mathrm{m}$;

- changement de la largeur de la bande avec conservation de la longueur d'onde central de la bande : bandes $7-15 \mu \mathrm{m}$ et $9-13 \mu \mathrm{m}$;

- décalage d'un des bords de la bande : bande 7 $14 \mu \mathrm{m}$ et $9-14 \mu \mathrm{m}$ (décalage de la limite des courtes longueurs d'ondes), bandes $8-13 \mu \mathrm{m}$ et $8-15 \mu \mathrm{m}$ (décalage de la limite des grandes longueurs d'ondes).

Pour toutes ces bandes nous utilisons une sensibilité spectrale uniforme rectangulaire comme pour la sensibilité spectrale S1 (Figure 2). Les différences de température de luminance ont été calculées pour les thermomètres infrarouges face à des sources d'émissivité constante (grise) (Figure 4).

Les différences de température de luminance obtenues avec des thermomètres infrarouges de bandes spectrales différentes de $\pm 1 \mu \mathrm{m}$ par rapport à la bande « idéale» 8 $14 \mu \mathrm{m}$ peuvent être supérieures à $1^{\circ} \mathrm{C}$ face à des sources dont 1'émissivité atteindrait 0,95 .
Tableau 1. Différence entre la température de luminance obtenue par les thermomètres infrarouges de sensibilités S2 à S5 face aux sources d'émissivités E1 à E8, et celle obtenue avec le thermomètre infrarouge de sensibilité $\mathrm{S} 1$ face aux mêmes sources.

\begin{tabular}{|c|c|c|c|c|c|}
\hline \multirow[t]{2}{*}{ Emissivité } & \multirow{2}{*}{$\begin{array}{l}\text { Tempéra- } \\
\text { ture de la } \\
\text { source, }{ }^{\circ} \mathrm{C}\end{array}$} & \multicolumn{4}{|c|}{$\begin{array}{l}\text { Différence des températures de luminance } \\
\qquad T_{\text {lum }}(\mathrm{Sx})-\mathrm{T}_{\text {lum }}(\mathrm{S} 1),{ }^{\circ} \mathrm{C}\end{array}$} \\
\hline & & $\mathrm{Sx}=\mathrm{S} 2$ & $\mathrm{Sx}=\mathrm{S} 3$ & $\mathrm{Sx}=\mathrm{S} 4$ & $\mathrm{Sx}=\mathrm{S} 5$ \\
\hline \multirow{6}{*}{ E1 } & -20 & 0.00 & 0.00 & 0.00 & 0.00 \\
\hline & 0 & 0.02 & -0.02 & 0.01 & -0.02 \\
\hline & 50 & 0.04 & -0.03 & 0.03 & -0.04 \\
\hline & 150 & 0.03 & -0.01 & 0.02 & -0.03 \\
\hline & 300 & -0.02 & 0.01 & -0.01 & 0.02 \\
\hline & 600 & 0.00 & 0.00 & 0.00 & 0.01 \\
\hline \multirow{6}{*}{ E2 } & -20 & -0.01 & 0.01 & -0.01 & 0.01 \\
\hline & 0 & 0.02 & -0.02 & 0.01 & -0.02 \\
\hline & 50 & 0.04 & -0.03 & 0.03 & -0.04 \\
\hline & 150 & 0.00 & 0.01 & 0.01 & -0.02 \\
\hline & 300 & -0.08 & 0.07 & -0.04 & 0.05 \\
\hline & 600 & -0.13 & 0.10 & -0.08 & 0.07 \\
\hline \multirow{6}{*}{ E3 } & -20 & -0.03 & 0.02 & -0.01 & 0.01 \\
\hline & 0 & 0.02 & -0.01 & 0.01 & -0.01 \\
\hline & 50 & 0.04 & -0.02 & 0.03 & -0.04 \\
\hline & 150 & -0.04 & 0.04 & 0.00 & -0.01 \\
\hline & 300 & -0.17 & 0.14 & -0.09 & 0.09 \\
\hline & 600 & -0.31 & 0.25 & -0.18 & 0.17 \\
\hline \multirow{6}{*}{ E4 } & -20 & -0.04 & 0.04 & -0.01 & 0.01 \\
\hline & 0 & 0.01 & -0.01 & 0.01 & -0.01 \\
\hline & 50 & 0.03 & -0.02 & 0.03 & -0.04 \\
\hline & 150 & -0.08 & 0.07 & -0.02 & 0.01 \\
\hline & 300 & -0.27 & 0.22 & -0.13 & 0.13 \\
\hline & 600 & -0.50 & 0.40 & -0.28 & 0.27 \\
\hline \multirow{6}{*}{ E5 } & -20 & -0.20 & 0.19 & -0.03 & 0.03 \\
\hline & 0 & -0.07 & 0.07 & 0.00 & 0.00 \\
\hline & 50 & 0.12 & -0.10 & 0.04 & -0.05 \\
\hline & 150 & 0.27 & -0.23 & 0.08 & -0.09 \\
\hline & 300 & 0.45 & -0.38 & 0.12 & -0.11 \\
\hline & 600 & 0.99 & -0.81 & 0.33 & -0.31 \\
\hline \multirow{6}{*}{ E6 } & -20 & 0.15 & -0.15 & 0.01 & -0.01 \\
\hline & 0 & 0.10 & -0.09 & 0.02 & -0.03 \\
\hline & 50 & -0.04 & 0.05 & 0.01 & -0.02 \\
\hline & 150 & -0.35 & 0.31 & -0.09 & 0.07 \\
\hline & 300 & -0.80 & 0.66 & -0.29 & 0.29 \\
\hline & 600 & -1.62 & 1.30 & -0.69 & 0.65 \\
\hline \multirow{6}{*}{ E7 } & -20 & -0.05 & 0.04 & -0.19 & 0.19 \\
\hline & 0 & 0.00 & 0.00 & -0.08 & 0.07 \\
\hline & 50 & 0.06 & -0.04 & 0.11 & -0.12 \\
\hline & 150 & 0.07 & -0.05 & 0.33 & -0.33 \\
\hline & 300 & 0.10 & -0.08 & 0.60 & -0.57 \\
\hline & 600 & 0.36 & -0.30 & 1.33 & -1.25 \\
\hline \multirow{6}{*}{ E8 } & -20 & -0.01 & 0.00 & 0.16 & -0.17 \\
\hline & 0 & 0.03 & -0.02 & 0.09 & -0.10 \\
\hline & 50 & 0.02 & -0.01 & -0.05 & 0.04 \\
\hline & 150 & -0.15 & 0.13 & -0.33 & 0.32 \\
\hline & 300 & -0.45 & 0.37 & -0.79 & 0.76 \\
\hline & 600 & -1.00 & 0.80 & -1.70 & 1.60 \\
\hline
\end{tabular}

Comme attendu, l'influence des courtes longueurs d'onde est plus importante. En effet, pour la plage de longueurs d'onde choisies (autour de $11 \mu \mathrm{m}$ ) et pour la plage de températures étudiées (de -20 à $600^{\circ} \mathrm{C}$ ), la dépendance de la luminance à la longueur d'onde est toujours plus importante pour les courtes longueurs d'onde (autrement dit, la dérivée de l'équation (4) par rapport à $\lambda$ est toujours plus élevée pour les courtes longueurs d'onde). 

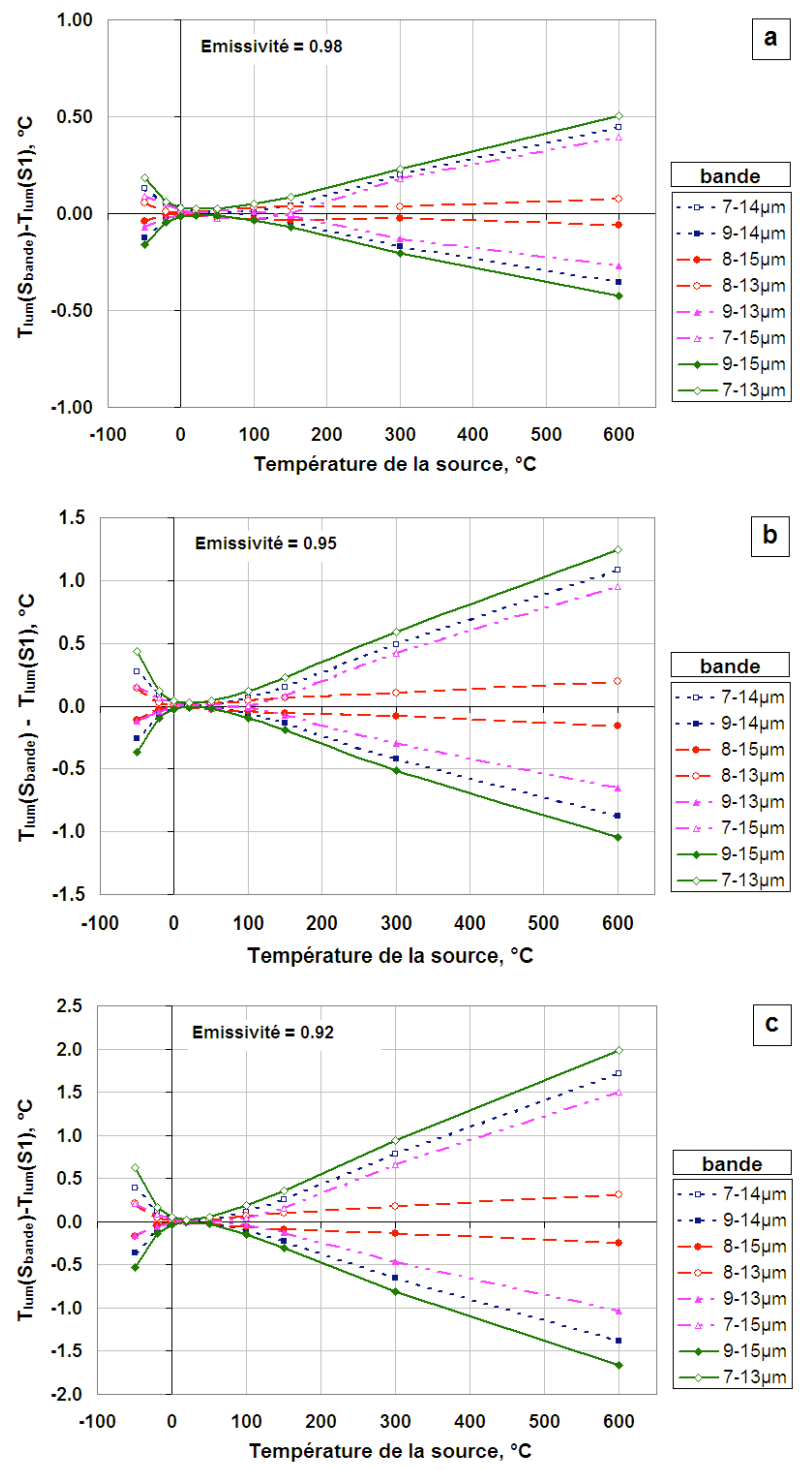

Figure 4. Différences de températures de luminance calculées pour les thermomètres infrarouges de bandes spectrales différentes face à des sources d'émissivités E2 $=0,98$ (a), E3 = 0,95 (b) et E4 =0,92 (c) en comparaison avec la température de luminance calculée pour le thermomètre infrarouge de bande spectrale $8-14 \mu \mathrm{m}$ (sensibilité spectrale S1).

Notons que pour ces calculs nous avons pris des sensibilités spectrales uniformes et des émissivités constantes. Dans ce cas, la différence des températures de luminance peut être obtenue avec la méthode décrite dans l'article [1], où la température de luminance est calculée en résolvant équation (3) de l'article. Nous avons effectué les mêmes calculs par cette méthode également. Les résultats obtenus par les deux méthodes sont en accord.

\section{Conclusion}

Les calculs décrits dans cet article ont montré que les écarts de température de luminance obtenue avec des thermomètres infrarouges de sensibilités spectrales différentes face à des sources d'émissivité non-unitaire et non-grise peuvent être supérieurs à $1^{\circ} \mathrm{C}$.
Les calculs d'influence de la bande spectrale du thermomètre infrarouge montre que le décalage de la limite des grandes longueurs d'onde est moins critique que le décalage de la limite des petites longueurs d'onde. L'effet de décalage de la limite les courtes longueurs d'ondes peut être supérieur à $1^{\circ} \mathrm{C}$.

Les résultats obtenus pour les courbes les plus basiques de sensibilités spectrales des thermomètres infrarouges et d'émissivités des sources mettent en évidence l'erreur qui peut être faite sur la température de luminance. Ces résultats peuvent être utiles pour établir un bilan d'incertitudes d'étalonnage des thermomètres infrarouges dans les laboratoires de transfert.

\section{Références}

1. P. Ridoux «Etalonnage au LNE des thermomètres infrarouges à émissivité fixe», 12e Congrès international de métrologie, Lyon, France (2005)

2. F. Sakuma, S. Hattori, «Establishing a practical temperature standard by using a narrow-band radiation thermometer with a silicon detector», in Temperature: Its Measurement and Control in Science and Industry, vol. 5, edited by J.F. Schooley, New York, AIP, 421-427 (1982)

3. P. Saunders, «General interpolation equations for the calibration of radiation thermometers», Metrologia, 34, 201-210 (1997)

4. J. Fischer et al., «CCT-WG5 on radiation thermometry, Uncertainty budgets for calibration of radiation thermometers below the silver point», Ver. 1.71, CIPM, CCT-WG5/docs-03-2008, Int. J. Thermophys. 29: 1066-1083 (2008) 